\title{
Assessment of design trade-offs for wireless power transfer on seawater
}

\author{
H. M. Santos*†, M. R. Pereira*, L. M. Pessoa*, Cândido Duarte*† and H. M. Salgado*† \\ ${ }^{*}$ INESC TEC \\ ${ }^{\dagger}$ Faculty of Engineering, University of Porto \\ Porto, Portugal
}

\begin{abstract}
In this work we propose a method for maximization of the efficiency of an underwater wireless power transfer system that has to cope with load changes, quality factor and coupling coefficient deviations. By means of 3D electromagnetic simulation and numerical computation, parameter analysis is accomplished using different compensation methods, namely series-series, series-parallel and parallel-parallel. Moreover, a linear load profile is assessed as a proof of concept applicable to more complex load behaviours. For this linear load variation a maximum measured average efficiency of $82 \%$ was obtained throughout the entire battery state of charge. Electronics and full system considerations are also presented. Finally, a good agreement between theoretical predictions of the proposed method, simulation assessment and measurement results was verified.

Index Terms-Underwater wireless power transfer, inductive coupling, magnetic coupling, AUV, seawater, coupled resonators, ABCD parameters, quality factor, coupling coefficient.
\end{abstract}

\section{INTRODUCTION}

Underwater sensor networks are becoming a standard practice for a broad number of activities. These include the inspection of subsea infrastructures, monitoring of seabed parameters and environmental control in open ocean oil extraction facilities. Such sensors can be installed permanently on an underwater structure or carried in a mobile platform. Fixed sensors are typically fed by batteries, as powering up these devices using cable connections can be excessively complex and costly.

Autonomous underwater vehicles (AUV) are becoming a standard practice in deep sea sensing due to their operational versatility. However, they are also limited by their battery duration. So a fully autonomous solution is not completely possible as a support needs to be present in order to recharge the batteries of AUVs. This, in turn, is also expensive and difficult to maintain due to the requirement of "wet-mate" connectors [1].

Underwater WPT is becoming the most attractive solution to recharge AUV batteries and ensure operational efficiencies. In [2], the authors showed the possibility to transfer energy through a conductive medium, such as seawater, with efficiencies up to approximately $81 \%$, for a gap of $15 \mathrm{~cm}$. They also concluded that optimized resonators for underwater WPT would be able to achieve higher efficiencies. In [3], the authors transferred power between a docking station and an AUV. However, alignment was critical as an efficiency of about $90 \%$ occurs for a gap of $2 \mathrm{~mm}$. This poses limitations on manoeuvrability of the AUV due to oscillations of the vehicle, while maintaining the required distance. As a result of these constraints, in [4] alignment cones were used to guide the
AUV in to position. Nevertheless, in the reported literature the design approach is not a optimum one since it does not account for load variations or other parameter fluctuations.

In this work a method for maximizing the efficiency of the wireless power transfer is proposed that accounts for AC load variation due to battery state of charge, coupling coefficient fluctuations as a result of docking maneuver and mechanical oscillations of the docking mechanism. Quality factor impact on average efficiency through all battery recharge process is also assessed and discussed.

This paper is organized as follows: section II presents the mathematical formulation for the efficiency of power transfer as a function of the parameters of interest, section III shows how the coupling coefficient can be measured either in simulation or experimentally, in section IV the design considerations and trade-offs are assessed, in section $\mathrm{V}$ overall system considerations are presented, section VI describes the measurement procedure and the relevant experimental results obtained, and section VII presents the relevant conclusions.

\section{POWER TRANSFER EFFICIENCY}

To calculate coil to coil efficiency we evaluate the ratio between power reaching the load $P_{\text {Load }}$ and power delivered to the network $P_{\text {Delivered }}$.

In order to easily obtain correct mathematical formulation, ABCD transmission parameters based on the generalized network of Fig. 1, [5], from which we write

$$
\left[\begin{array}{l}
V_{1} \\
I_{1}
\end{array}\right]=\left[\begin{array}{ll}
A & B \\
C & D
\end{array}\right]\left[\begin{array}{l}
V_{2} \\
I_{2}
\end{array}\right]
$$

where $V_{1}$ and $V_{2}$ are the voltages of port 1 and port 2 and the corresponding currents entering and leaving the network are $I_{1}$ and $I_{2}$, , respectively. The input impedance $Z_{i n}$ is derived as

$$
Z_{\text {in }}=\frac{V_{1}}{I_{1}}=\frac{A+B / R_{L}}{C+D / R_{L}}
$$

whereas the voltage gain, $G$, is given by

$$
G=\frac{V_{2}}{V_{1}}=\frac{1}{A+B / R_{L}}
$$

Using equations (2) and (3), we write the network power transfer efficiency as

$$
\eta=\frac{P_{\text {Load }}}{P_{\text {Delivered }}}=\frac{1 / 2 \times|G|^{2}\left|V_{1}\right|^{2} / R_{L}}{\Re\left(1 / 2 \times\left|V_{1}\right|^{2} / \overline{Z_{\text {in }}}\right)}=\frac{|G|^{2} / R_{L}}{\Re\left(1 / \overline{Z_{\text {in }}}\right)}
$$




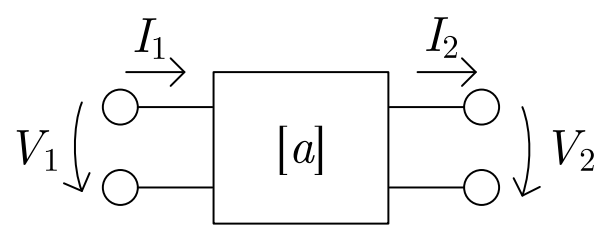

Fig. 1. ABCD generic network.

in which $\overline{Z_{i n}}$ represents the complex conjugate of the input impedance. By denoting $\mathbf{A}_{\mathbf{L}}$ as the transmission matrix for a series connected inductor with a parasitic series resistance and $\mathbf{A}_{\mathbf{K}}$ the $\mathrm{ABCD}$ matrix for a $\mathrm{K}$ impedance inverter, we have

$$
\mathbf{A}_{\mathbf{L}}=\left[\begin{array}{cc}
1 & j X_{L}+X_{L} / Q \\
0 & 1
\end{array}\right]
$$

and

$$
\mathbf{A}_{\mathbf{K}}=\left[\begin{array}{cc}
0 & -j k X_{L} \\
-\frac{j}{k X_{L}} & 0
\end{array}\right]
$$

where $k$ is the coupling coefficient and $Q$ the inductor quality factor; $X_{L} / Q$ represents the series resistance of the coil. Employing equations (5) and (6) the ABCD matrix of the equivalent loosely coupled transformer $\mathbf{A}_{\text {Transf }}$, shown Fig. 2 , can be expressed as

$$
\begin{aligned}
\mathbf{A}_{\text {Transf }} & =A_{L} \times A_{K} \times A_{L}= \\
& =\left[\begin{array}{cc}
0 & -j k X_{L} \\
-\frac{j}{k X_{L}} & \frac{1}{k}-\frac{j}{k Q}
\end{array}\right]
\end{aligned}
$$

To compensate the transformer with the introduction of a resonance, we connect series or parallel capacitors in either side of the loosely coupled transformer. The ABCD matrix of a shunt connected capacitor is

$$
\mathbf{A}_{\text {Cshunt }}=\left[\begin{array}{cc}
1 & 0 \\
\frac{j}{X_{C}} & 1
\end{array}\right]
$$

and the transmission matrix of a series connected capacitance

$$
\mathbf{A}_{\text {Cseries }}=\left[\begin{array}{cc}
1 & -j X_{C} \\
0 & 1
\end{array}\right] .
$$

By multiplication of the matrices (8)/(9) and (7) and (8)/(9), the ABCD matrix of the compensated system, parallel/seriesparallel/series, can be obtained. The parallel-parallel system is written as

$$
\mathbf{A}_{\mathbf{P A R}}=\mathbf{A}_{\text {Cshunt }} \times \mathbf{A}_{\text {Transf }} \times \mathbf{A}_{\mathbf{C s h u n t}},
$$

the series-parallel compensation is

$$
\mathbf{A}_{\text {SP }}=\mathbf{A}_{\text {Cseries }} \times \mathbf{A}_{\text {Transf }} \times \mathbf{A}_{\text {Cshunt }},
$$

and the series-series resonance becomes

$$
\mathbf{A}_{\text {SER }}=\mathbf{A}_{\text {Cseries }} \times \mathbf{A}_{\text {Transf }} \times \mathbf{A}_{\text {Cseries }} .
$$

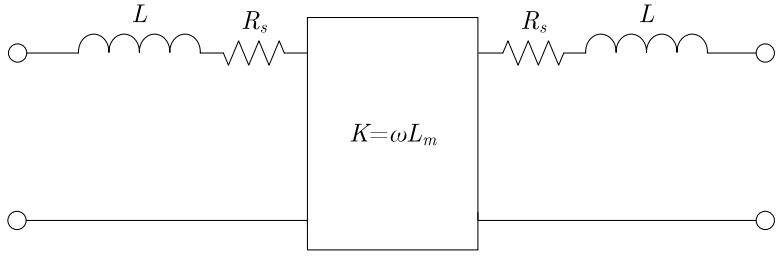

Fig. 2. Loosely coupled transformer equivalent circuit.

Using the $\mathrm{ABCD}$ matrices of equations (10), (11), (12) and combining them on equation (4), at the resonant frequency where $X_{C}=X_{L}$, the efficiency of a series-series compensated system is given by

$$
\eta_{s e r}=\frac{Q^{3} k^{2} X_{L}^{2} R_{L}}{\left(Q^{2} X_{L}^{2} k^{2}+Q X_{L} R_{L}+X_{L}^{2}\right)\left(Q R_{L}+X_{L}\right)}
$$

whereas for the series-parallel or parallel-parallel resonated transformer the efficiency is

$$
\begin{aligned}
\eta_{\text {par }}= & Q^{3} X_{L}^{3} k^{2} R_{L} /\left[\left(Q^{2}+1\right) X_{L}^{4}+2 Q X_{L}^{3} R_{L}+\right. \\
& +\left(k^{2} X_{L}^{2} Q^{2}+R_{L}^{2}\right) X_{L}^{2}+Q^{3} X_{L}^{3} k^{2} R_{L}+ \\
& \left.+Q^{2} k^{2} X_{L}^{2} R_{L}^{2}\right]
\end{aligned}
$$

\section{Coupling COEFFicient}

According to [6], understanding the relation between the coupling coefficient and certain physical parameters, such as the distance between inductors, is of fundamental importance. This is how the design of a WPT systems is usually performed. In this paper we obtain an analytic expression for the coupling factor and show how it is influenced by the distance between spiral inductors. This has the advantage of allowing the UWPT system designer to easily obtain the coupling factor behaviour and take this into consideration in the optimization process.

\section{A. Theory}

The coupling coefficient depends on the physical parameters of the system which in turn determine the location of the resonances. In this section, using mode theory, we will derive the relation between the coupling coefficient, $k$, and the resonances $\omega_{1}$ and $\omega_{2}$, which upon their measurement enables one to indirectly assess the coupling coefficient.

Mode theory requires the use of high values of the impedance at the generator and load, so that the unloaded network becomes the circuit represented in Fig. 3. The two

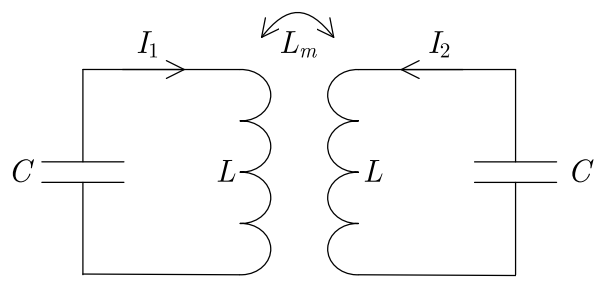

Fig. 3. Coupled resonators circuit. 
unloaded inductively coupled LC resonators in this Figure can be described by

$$
\left[\begin{array}{cc}
j\left(\omega L-\frac{1}{\omega C}\right) & j \omega L_{m} \\
j \omega L_{m} & j\left(\omega L-\frac{1}{\omega C}\right)
\end{array}\right]\left[\begin{array}{l}
I_{1} \\
I_{2}
\end{array}\right]=\left[\begin{array}{l}
0 \\
0
\end{array}\right],
$$

which is an homogeneous system of equations. Solving for this circuit to have a non-trivial solution yields the determinant

$$
\left|\begin{array}{cc}
j\left(\omega L-\frac{1}{\omega C}\right) & j \omega L_{m} \\
j \omega L_{m} & j\left(\omega L-\frac{1}{\omega C}\right)
\end{array}\right|=0
$$

from which the two resonant frequencies of the system are obtained

$$
\omega_{1}=\frac{1}{\sqrt{\left(L-L_{m}\right) C}}
$$

and

$$
\omega_{2}=\frac{1}{\sqrt{\left(L+L_{m}\right) C}} .
$$

Combining equations (17), (18) and knowing that $L_{m}=k L$, we can write

$$
k=\frac{\left|\omega_{1}^{2}-\omega_{2}^{2}\right|}{\omega_{1}^{2}+\omega_{2}^{2}}
$$

which allows us to obtain the coupling coefficient by determining the two resonant frequencies either by simulation or experimentally.

\section{B. Simulation Assessment}

Simulation was performed in HyperWorks FEKO, a 3D simulation based on the method of moments (MoM). The corresponding model is depicted in Fig. 4. The surrounding medium was set as salt water with a conductivity of $\sigma=4 \mathrm{~S} / \mathrm{m}$ and relative permittivity of $\epsilon_{r}=81$ [7]. The inner diameters of 5 and 15 turn inductors were swept in the range of $5 \leq D_{\text {inner }} \leq 7 \mathrm{~cm}$ for the former case and $5 \leq D_{\text {inner }} \leq 9 \mathrm{~cm}$ for the latter. This procedure allows us to understand the dependence of the coupling coefficient on the turn number, $N$, and inner diameter, $D_{\text {inner }}$. The outer diameter $\left(D_{\text {outer }}\right)$ was also given two values of $16 \mathrm{~cm}$ and $10 \mathrm{~cm}$, to analyse its impact on $k$. Additionally, to assess the coupling factor changes, distance between inductors was swept in the range of $1 \leq s \leq 10 \mathrm{~cm}$.

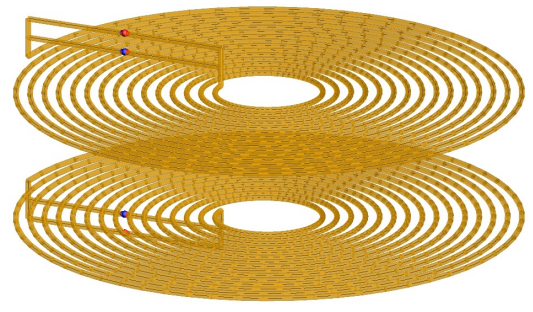

Fig. 4. FEKO simulation model.
Figs. 5 and 6, show the coupling coefficient change with the distance between inductors, for 5 and 15 turns inductors, respectively, having an outer diameter of $D_{\text {outer }}=16 \mathrm{~cm}$, for several inner diameters. From these results, it is seen almost no dependence of the coupling factor on the number of turns and the inner diameter.

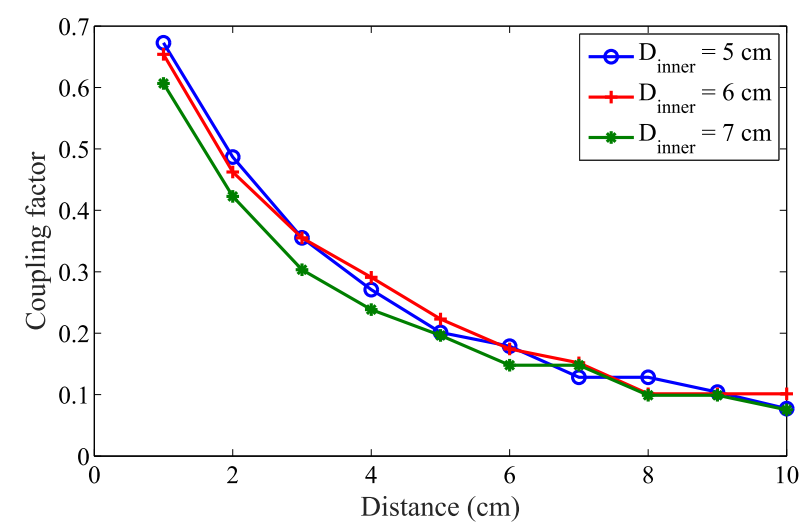

Fig. 5. Coupling coefficient as a function of distance for 5 turn inductors with different inner diameters.

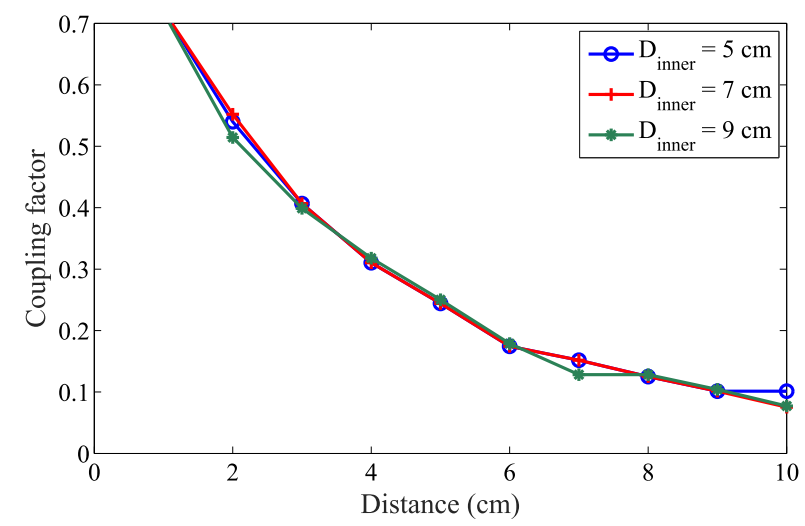

Fig. 6. Coupling coefficient as a function of distance for 15 turn inductor different inner diameters.

Fig. 7 plots the coupling factor as a function of the distance, $s$, for two inductors with outer diameters of $10 \mathrm{~cm}$ and $16 \mathrm{~cm}$. We can conclude that $k$ depends mostly on the outer diameter of the inductor and on the distance between spirals. Furthermore, it is possible to perceive that physically larger inductors are desirable for greater coupling coefficients.

A further analysis of the results, leads us to conclude that the evolution of $k$ as distance increases is similar to an exponential decay. For this reason, using MathWorks MATLAB we employed a curve fitting procedure to the generic exponential decay equation

$$
k=C_{1} \times \exp \left(-\frac{C_{2} \cdot s}{D_{\text {outer }}}\right)
$$

where $s$ is the spacing between coils, $C_{1}$ and $C_{2}$ are constants whose values are obtained from the curve fitting procedure. 


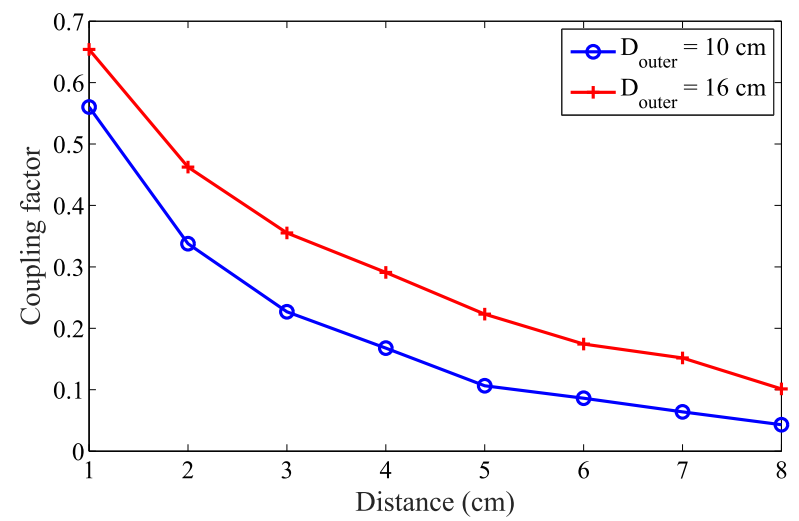

Fig. 7. Coupling coefficient as a function of distance for 5 turn inductors with different outer diameters.

Adjusting these constants for the two study cases presented in Fig. 7 , yields $C_{1} \approx 0.8$ and $C_{2} \approx 4$. In Fig. 8 the simulation results with their respective fitted curves are shown and a good agreement can be verified in both cases.

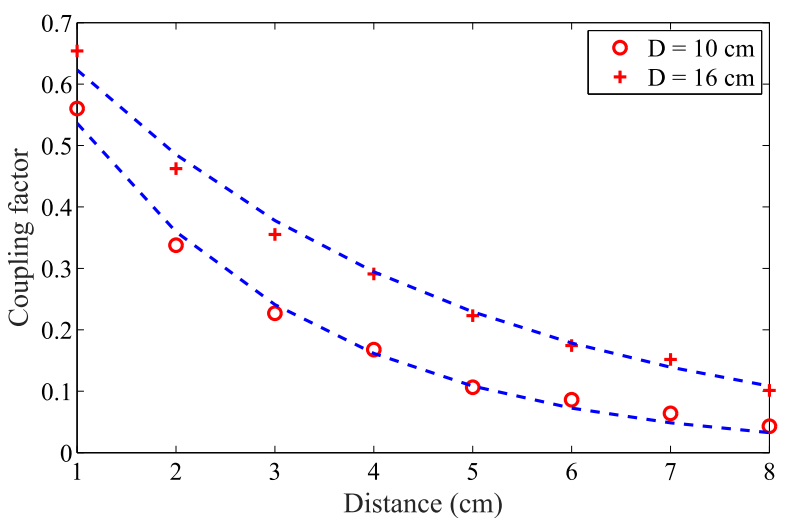

Fig. 8. Coupling coefficient as a function of distance for 5 turn inductors with different outer diameters and fitted empirical curves.

\section{VARIABle LoAd Design CONSIDERATIONS}

In the analysis of WPT systems usually either fixed or optimal loads are considered for a given steady regime. However, for the case of underwater WPT, in the specific scenario of recharging the batteries of AUVs, such regime is absent. When these vehicles approach the docking station, the coupling coefficient is changing. Even when they are docked, due to oscillations and misalignments, system state fluctuations should be accounted for optimal performance.

In our specific case, we use $16 \mathrm{~cm}$ spiral inductors due to space restrictions of the MARES AUV [8]. The gap between coils is estimated to be $4 \mathrm{~cm}$, which after applying equation (20) gives a nominal coupling factor of $k=0.294$. Additionally, according to [9] and [10], quality factors around 100 were achieved for helical inductors and spiral coils, respectively. For this reason we use a nominal value for the quality factor of the $\mathrm{LC}$ resonator of $Q=100$.
To assess the AC load variability we assume an unknown rectifier connected to a battery which presents a fluctuating load to the transformer secondary winding. Such AC load variations occur as the battery state of charge (SoC) from this point onwards referred to by $\psi$, changes. As an arbitrary example to assess the load influence on efficiency, we assume that the receiver resistance varies linearly with $\psi$ between 1 and $50 \Omega$, according to equation

$$
R_{L}=1+50 \psi
$$

Substituting equation (21) in equations (13) and (14) gives the efficiency as a function of the battery state of charge for a series-series resonated transformer $\eta_{\text {ser }}(\psi)$ and for parallelparallel/series-parallel compensation $\eta_{\text {par }}(\psi)$, respectively. To achieve maximum performance throughout all the charging process, we take the average value of the efficiency during recharge and optimize it. We can choose coil reactance $X_{L}$ and optimize $Q$ to minimize losses. However, $k$ can also suffer variations due to a non-ideal docking process.

In Figs. 9 and 10 the average coil to coil efficiency as a function of coil reactance is plotted, for a series-series compensated transformer, with the $Q$ and $k$ factors as parameters, respectively. It can be seen in both figures that either $Q$ or $k$ below their nominal values will decrease the average efficiency, regardless of coil reactance. On the contrary, higher $Q$ values increase average coil to coil efficiency, as resistive losses are smaller on the resonators. Moreover, a maximum of the average efficiency is observed for a coil reactance of $X_{L}=66 \Omega$. This allows us to conclude that if a linear dependence between load and SoC exists, an optimal coil reactance maximizes average efficiency.

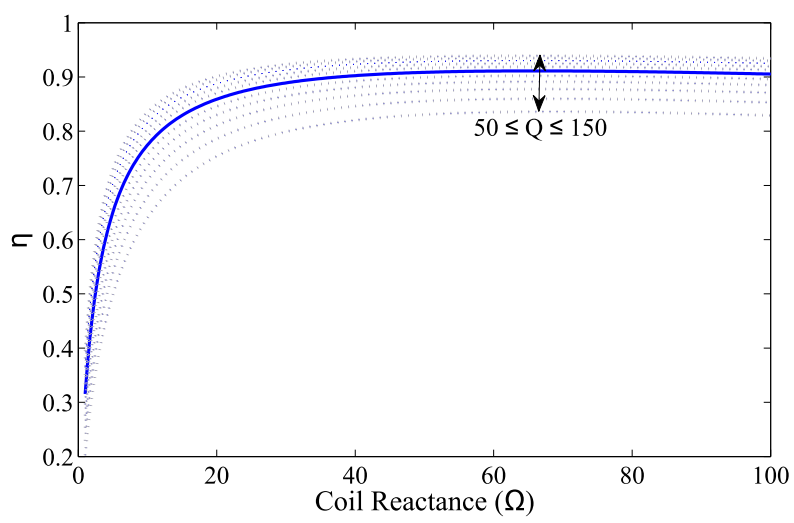

Fig. 9. Average coil to coil efficiency as a function of coil reactance and $Q$ variation for series-series compensation.

As seen in Figs. 11 and 12 the average efficiency fluctuation with design parameters is shown. It can be seen that a similar but more pronounced behaviour exists in relation to series-series compensation scheme. Identical efficiencies are achieved and just as in the previous case, $Q$ increments lead to higher efficiency. Additionally lowering $k$ yields smaller efficiencies as expected. Once again, it is possible to 


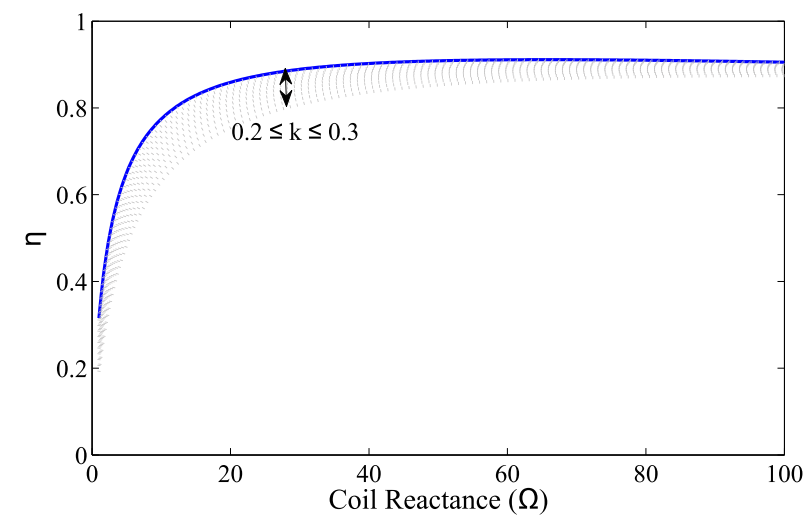

Fig. 10. Average coil to coil efficiency as a function of coil reactance and $k$ for series-series compensation.

conclude that an optimal reactance value exists, for seriesparallel/parallel-parallel configurations. For nominal $Q$ and $k$ values the optimal value of the coil reactance that maximizes the average efficiency is $X_{L} \approx 5.3 \Omega$, considering the linear behaviour of the AC load formerly mentioned.

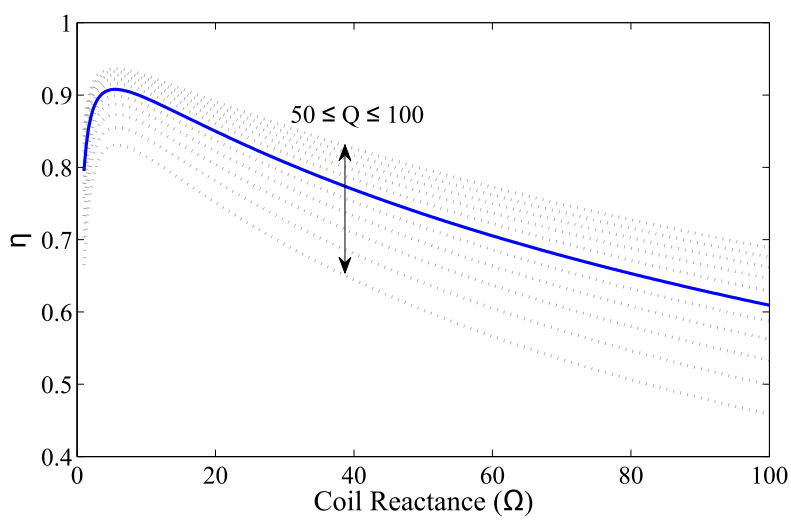

Fig. 11. Average coil to coil efficiency as a function of coil reactance and $Q$ variation for parallel-parallel/series-parallel compensation.

From the previous results we can conclude that $Q$ changes do not affect much the optimal coil reactance value. On the other hand, $k$ variations will have a considerable impact on deviating the optimal coil reactance from the nominal value considered in normal conditions. Considering this specific load variation as a function of the $\mathrm{SoC}$, we can perceive that series-parallel/parallel-parallel configurations demand smaller coil reactance values to achieve similar efficiencies as in the series-series compensation scheme. However, parallel-parallel or series-parallel compensated WPT systems are more sensitive to changes in $Q$ and $k$, for optimum $X_{L}$. Another load behaviour other the one considered will yield different results and the present procedure would have to be re-evaluated if maximum possible average efficiencies are desired. $X_{L}$ can be adjusted either by changing the resonant frequency or the inductance value. However, this will have a direct impact

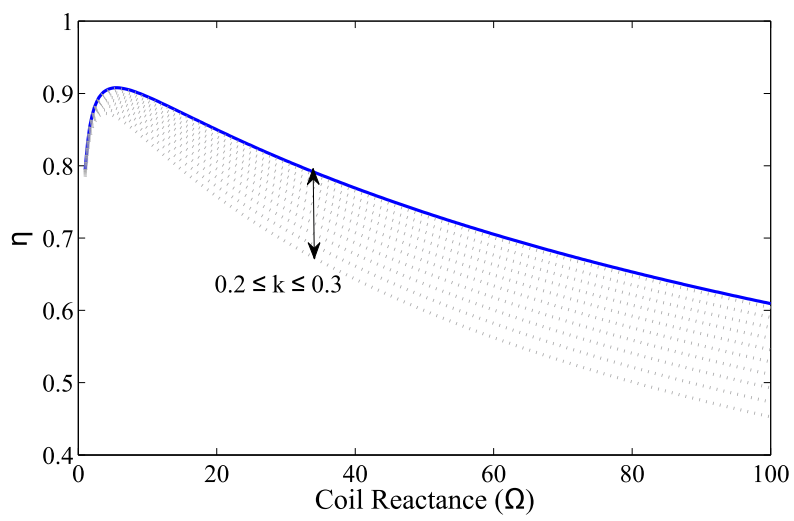

Fig. 12. Average coil to coil efficiency as a function of coil reactance and $k$ for parallel-parallel/series-parallel compensation.

on the $Q$ factor and consequently on the average efficiency, which leads to a complicated dependence of all the parameters involved.

Analytical derivations were attempted for the load variations being studied, that led in the end to transcendental equations. Nonetheless, assuming that an analytical model of the $\mathrm{AC}$ load presented by the rectifier and battery assembly exists, an optimum $X_{L}$ value may be encountered by solving

$$
\frac{\mathrm{d}}{\mathrm{d} X_{L}}\left[\int_{0}^{1} \eta(\psi) \mathrm{d} \psi\right]=0
$$

which actually is equivalent to equating to zero the derivative of average efficiency with respect to $X_{L}$, which in turn is the same as finding the coil reactance value that maximizes coil to coil efficiency throughout the entire recharging process.

Additionally, assuming that only discrete measurements of the AC load versus the SoC exist, the optimum reactance can be found by taking the derivative of the arithmetic mean of all efficiencies with respect to $X_{L}$

$$
\frac{\mathrm{d}}{\mathrm{d} X_{L}}\left[\frac{1}{n} \sum_{i=1}^{n}\left(\eta_{i}\right)\right]=0
$$

where $n$ is the number of measurements and $\eta_{i}$ is the efficiency equation obtained by replacing $R_{L}$ with each rectifier input resistance measurement, $R_{i}$.

\section{CONSIDERATIONS ABOUT THE COMPlete SYSTEM}

For the full implementation of the battery recharge system in underwater WPT, additional circuitry is required. A power stage should drive the primary coil and a rectifier is required at the battery side. The most straightforward approach for driving the coils is to use a half-bridge inverter in series-series or series-parallel configurations [12]. In spite of the frequencydependent power losses due to the output capacitance of the power devices, a class-D inverter avoids significant voltage peaks above power supply, which may pose reliability issues to the transistors. Other driver configurations such as those based on current-mode topologies induce considerable voltage 
stress [13], but can make use of network symmetry when applicable. At the load side, the system comprises a rectifier usually employing diodes supporting elevated reversed peak voltage, and a voltage regulation system that controls the charge of the battery, herein represented by $R_{L}$ (that naturally changes along time).

Fig. 13 depicts a WPT complete system comprising a class-D inverter and a simple rectifier. The coupling coils are represented by means of their "tee" model, comprising auto-inductances $L_{1}$ and $L_{2}$, and mutual inductance $L_{m}$. The capacitors $C_{1}$ and $C_{2}$ are the passive elements added to establish resonance (series or parallel) with the coupling coils at the desired switching frequency. The driver is excited by complementary signals at high- and low-side terminal $\left(v_{h s}\right.$ and $v_{l s}$ respectively in Fig. 13), having some dead-time to prevent switching losses due to shoot-through and losses due to body-diode conduction.



Fig. 13. Series-parallel resonance topology.

The efficiency profile is significantly different in seriesseries and series-parallel topologies - see Fig. 14(a). Although one can design a driver in which the peak has nearly the same value, they occur at different load ranges. Series-series tend to have better efficiencies at reduced load regimes, whereas when the load is subject to a wider variation at higher values, the efficiency in series-parallel is less affected. Furthermore, there is a tradeoff between power delivered (or output power) and efficiency. Hence, in some cases both performance characteristics have to be considered. When maximum power is required, the series-series topology gives superior power delivery in spite of the decreasing efficiency with the load - see Fig. 14(b).

\section{RESULTS}

From the theoretical results obtained in section IV, we conclude that for the load behaviour under analysis, achieving optimal average efficiency using parallel-parallel compensation demands a coil reactance of $X_{L}=5.3 \Omega$.

Two 5 turn spiral coils were assembled and mounted on the measurement setup as shown in Fig. 15. Using an LCR meter a $4.46 \mu \mathrm{H}$ inductance was measured. This reactance is optimum at the frequency $\omega=X_{L} / L \approx 1.188 \mathrm{Mrad} / \mathrm{s}$ or equivalently to $f=\omega /(2 \pi) \approx 189 \mathrm{kHz}$. In order to obtain this resonant frequency, we calculate the required capacitance as being $C=\frac{1}{\omega^{2} L} \approx 159 \mathrm{nF}$. Due to component availability, however, in practice $150 \mathrm{nF}$ capacitances were used to parallel resonate both inductors, which resulted in a resonant frequency of $f_{0}=$

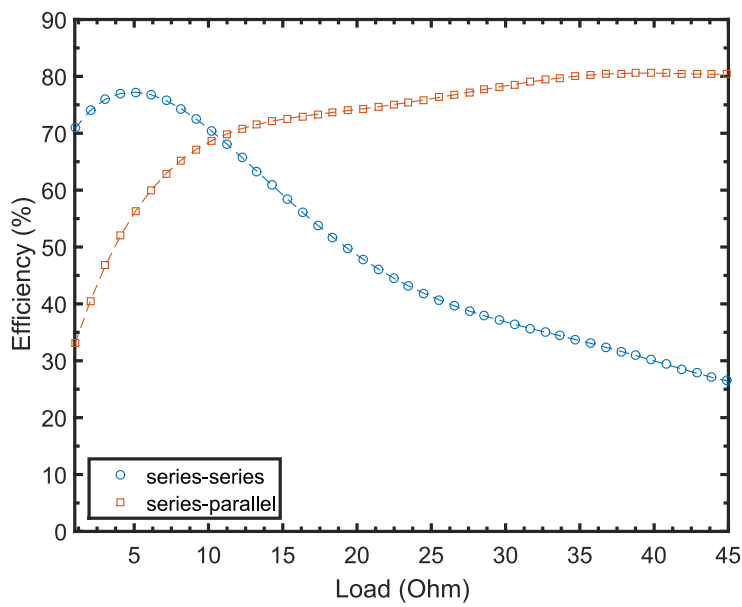

(a)

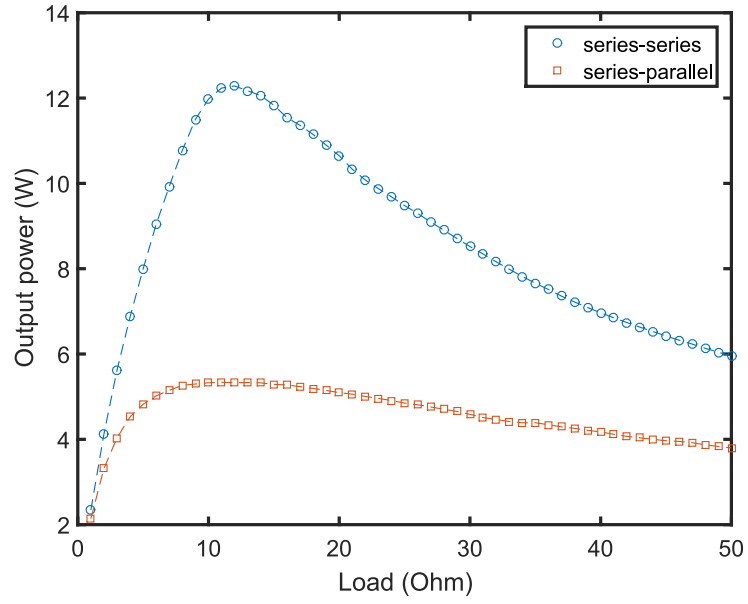

(b)

Fig. 14. (a) Efficiencies and (b) power output of series-parallel and seriesparallel resonance topologies.

$194.6 \mathrm{kHz}$ and a coil reactance of $X_{L}=2 \pi f_{0} L \approx 5.45 \Omega$. At $f=194.6 \mathrm{kHz}$ the quality factor of a single inductor was measured as $Q=68.4$, using the LCR meter. After connecting the parallel capacitor to achieve resonance, a quality factor of $Q=58.4$ was measured indirectly from the input impedance as described in [10], which is lower than the expected as capacitors have $Q=400$ [11]. By unloading the resonators with series capacitors as explained in subsection III-A, we measured two $\left|S_{21}\right|$ peaks on the VNA at $f_{1}=122.4 \mathrm{kHz}$ and $f_{2}=158.1 \mathrm{kHz}$, which resulted in a coupling coefficient of $k \approx 0.25$ for a gap of $4 \mathrm{~cm}$. Simulation results using FEKO, gave values of $k=0.28, Q=65$ and $X_{L}=5.17 \Omega$.

Employing the efficiency equation for a parallel-parallel system as a function of SoC (14), yields the plot of Fig. 16 where simulated and measured efficiencies are shown. A good agreement can be seen, with an obvious degradation of the measured results in relation to the simulated ones. Such performance decrease occurs due to a lower quality factor of the fabricated resonator as non-ideal capacitors are used. On the other hand, in simulation ideal capacitors of $150 \mathrm{nF}$ 


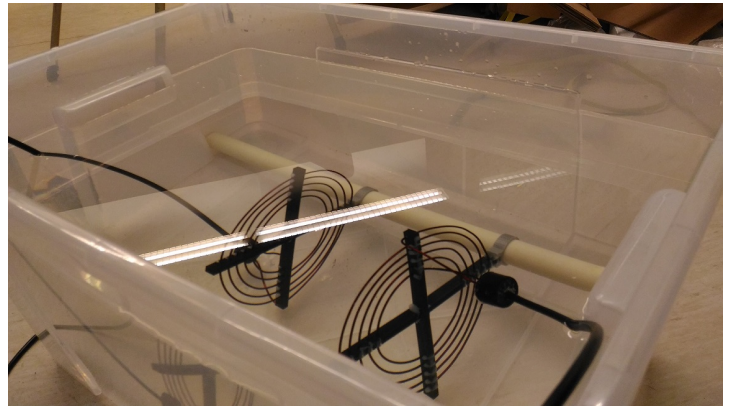

Fig. 15. 5 turn spiral inductors inside water tank.

were used which resulted in a higher $Q$. A measured average efficiency of $\widehat{\eta}_{\text {meas }}=82.13 \%$ and a simulated average efficiency of $\widehat{\eta}_{\text {sim }}=85.98 \%$ were obtained, which shows approximately a $4 \%$ deviation between ideal and measured behaviour.

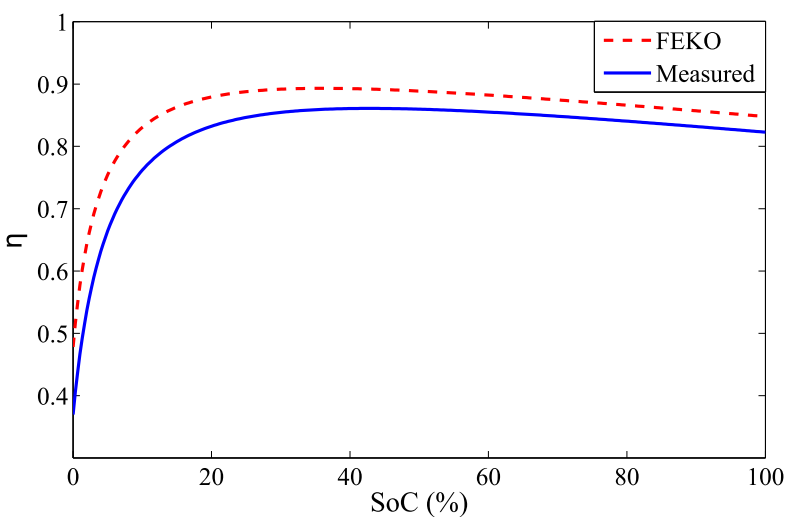

Fig. 16. Simulated and measured efficiencies as a function of battery state of charge for linear behaviour example under analysis.

\section{CONCLUSION}

In this work a power transfer efficiency maximization method was provided, so that load variations which might occur in the rectifier and battery are accounted for. Variations of quality factor and coupling coefficient that are prone to exist due to lossy capacitors and non-ideal docking maneuver, respectively, were also considered in optimizing efficiency. To contemplate load behaviour, efficiency as a function of the battery state of charge was evaluated. To achieve optimal performance, average efficiency along the overall load variation was maximized for three types of compensations, namely series-series, series-parallel and parallel-parallel. A measured maximum average efficiency of $82.13 \%$ was obtained in parallel-parallel configuration for an hypothetical linear load variation between 1 and $50 \Omega$. We also concluded that other load profiles will yield different results. Nevertheless, this procedure was proposed to optimize efficiency for any load profile either from an analytical formulation or discrete measurements.

\section{ACKNOWLEDGEMENT}

This work has been developed as part of the project ENDURE- Enabling Long Term Deployments of Underwater Robotic Platforms in Remote Oceanic Locations, under the EEA Grant No. PT02-Aviso4-0015, and TECSEA research infrastructure. We also acknowledge support from he TEC4SEA research infrastructure (www.tec4sea.com), and COST action IC1301 - WIPE.

\section{REFERENCES}

[1] T. Kojiya, F. Sato, H. Matsuki, and T. Sato, "Automatic power supply system to underwater vehicles utilizing non-contacting technology," in OCEANS '04. MTTS/IEEE TECHNO-OCEAN '04, vol. 4, Nov 2004, pp. 2341-2345 Vol.4.

[2] M. Kesler and C. McCarthy, "Highly resonant wireless power transfer in subsea applications," in WiTricity white paper, 2013.

[3] Z.-s. Li, D.-j. Li, L. Lin, and Y. Chen, "Design considerations for electromagnetic couplers in contactless power transmission systems for deep-sea applications," Journal of Zhejiang University SCIENCE $C$, vol. 11, no. 10, pp. 824-834, 2010. [Online]. Available: http://dx.doi.org/10.1631/jzus.C0910711

[4] C.-j. Y. Jian-guang Shi, De-jun Li, "Design and analysis of an underwater inductive coupling power transfer system for autonomous underwater vehicle docking applications," Journal of Zhejiang University Science C, vol. 15, no. 1, pp. 51-62, 2014.

[5] D. Pozar, Microwave Engineering, 4th Edition. Wiley, 2011.

[6] L. Chen, S. Liu, Y. C. Zhou, and T. J. Cui, "An optimizable circuit structure for high-efficiency wireless power transfer," IEEE Transactions on Industrial Electronics, vol. 60, no. 1, pp. 339-349, Jan 2013.

[7] E. A. Karagianni, "Electromagnetic waves under sea: bow-tie antennas design for wi-fi underwater communications," in Progress In Electromagnetics Research $M$, vol. 41, 2015, pp. 189-198.

[8] N. A. Cruz and A. C. Matos, "The MARES AUV, a Modular Autonomous Robot for Environment Sampling," in OCEANS 2008, Sept 2008, pp. 1-6.

[9] J. Kuipers, H. Bruning, D. Yntema, S. Bakker, and H. Rijnaarts, "Selfcapacitance and resistive losses of saline-water-filled inductors," IEEE Transactions on Industrial Electronics, vol. 61, no. 5, pp. 2356-2361, May 2014.

[10] H. M. Santos, M. R. Pereira, L. M. Pessoa, and H. M. Salgado, "Design and optimization of air core spiral resonators for magnetic coupling wireless power transfer on seawater," in 2016 IEEE Wireless Power Transfer Conference (WPTC), May 2016, pp. 1-4.

[11] AC and Pulse Double Metallized Polypropylene Film Capacitors, Vishay, 32013.

[12] F. Gonçalves, C. Duarte, and L. M. Pessoa, "A novel circuit topology for underwater wireless power transfer," in International Conference on Systems Informatics, Modelling and Simulation, Riga, Latvia, Jun 2016, pp. 181-186.

[13] D. Oliveira, C. Duarte, V. G. Tavares, and P. G. de Oliveira, "Design of a current-mode class-D power amplifier in RF-CMOS," in Proceedings of the XXIV Conference on Design of Circuits and Integrated Systems (DCIS'2009), Zaragoza, Spain, Nov 2009, pp. 418-422. 